\title{
Combined interhemispheric and pterional approach for ACOM and left MCA aneurysms
}

Satoshi Kiyofuji, M.D., Tomohiro Inoue, M.D., Akira Tamura, M.D., Ph.D., and Isamu Saito, M.D., Ph. D.

Department of Neurosurgery, Fuji Brain Institute and Hospital, Fujinomiya City, Shizuoka, Japan

This video demonstrates combined two separate craniotomies for two difficult unruptured cerebral aneurysms. The anterior communicating artery (ACOM) aneurysm existed at a high position, projected posteriorly, and thus necessitated an interhemispheric approach. Left middle cerebral artery (MCA) aneurysm with complex figure was treated through a separate pterional approach. Meticulous micro-cisternal opening under high magnification enabled safe and effective exposure of both aneurysms with minimal brain retraction, which alleviated brain damage as shown in postoperative images.

The video can be found here: http://youtu.be/mBYsaAVekCA.

KEY WORDS unruptured cerebral aneurysm; clipping; interhemispheric approach; pterional approach 